\title{
Stochastic Model Predictive Control for Building Climate Control
}

\author{
Frauke Oldewurtel, Member, IEEE, Colin Neil Jones, Member, IEEE, \\ Alessandra Parisio, Member, IEEE, and Manfred Morari, Fellow, IEEE
}

\begin{abstract}
In this brief paper, a Stochastic Model Predictive Control formulation tractable for large-scale systems is developed. The proposed formulation combines the use of Affine Disturbance Feedback, a formulation successfully applied in robust control, with a deterministic reformulation of chance constraints. A novel approximation of the resulting stochastic finite horizon optimal control problem targeted at building climate control is introduced to ensure computational tractability. This work provides a systematic approach toward finding a control formulation which is shown to be useful for the application domain of building climate control. The analysis follows two steps: 1) a small-scale example reflecting the basic behavior of a building, but being simple enough for providing insight into the behavior of the considered approaches, is used to choose a suitable formulation; and 2) the chosen formulation is then further analyzed on a large-scale example from the project OptiControl, where people from industry and other research institutions worked together to create building models for realistic controller comparison. The proposed Stochastic Model Predictive Control formulation is compared with a theoretical benchmark and shown to outperform current control practice for buildings.
\end{abstract}

Index Terms-Affine disturbance feedback (ADF), building climate control, chance constraints, Stochastic model predictive control (SMPC).

\section{INTRODUCTION}

$\mathbf{T}$ HIS brief paper is concerned with solving a Model Predictive Control (MPC) problem for the class of discretetime linear systems subject to stochastic disturbances. The aim is to provide a method for efficiently finding control policies given a set of polytopic input constraints and chance constraints on the state, which is computationally tractable to be applicable to large-scale systems.

One example of a control problem, which naturally leads to chance constraints and which is the primary motivation for this brief paper, originates from building climate control. In European building standards, it is required that the room temperature is kept within a comfort range with a predefined probability [1]. The control problem is to minimize energy while satisfying this chance constraint. MPC for building

Manuscript received April 20, 2012; revised May 2, 2013; accepted June 21, 2013. Manuscript received in final form July 1, 2013. Recommended by Associate Editor E. Kerrigan.

F. Oldewurtel is with the Power Systems Laboratory, ETH Zurich, Zurich 8092, Switzerland (e-mail: oldewurtel@eeh.ee.ethz.ch).

C. N. Jones is with the Automatic Control Laboratory, EPFL Lausanne, Lausanne CH-1015, Switzerland (e-mail: colin.jones@epfl.ch).

A. Parisio is with the Automatic Control Laboratory, KTH Stockholm, Stockholm SE-100 44, Sweden (e-mail: parisio@kth.se).

M. Morari is with the Automatic Control Laboratory, ETH Zurich, Zurich 8092, Switzerland (e-mail: morari@control.ee.ethz.ch).

Color versions of one or more of the figures in this paper are available online at http://ieeexplore.iee.org.

Digital Object Identifier 10.1109/TCST.2013.2272178 climate control using weather and occupancy predictions has been addressed in several works, see [2]-[10] or the web site of the OptiControl project [11] and the references therein.

This brief paper provides a systematic approach to address the uncertainty in weather predictions, formulate chance constraints, and obtain a suitable control formulation for the building application domain. A deterministic reformulation of the chance constraints is combined with affine disturbance feedback, a parameterization of the control inputs, to obtain a tractable formulation of stochastic MPC, applicable also to large-scale systems such as buildings. By using affine disturbance feedback and by exploiting the fact that comfort violations are allowed from time to time with the chance constraints, the conservatism of traditional robust solutions is reduced. The presented material is an extension to the ideas proposed in [12] and offers a comparison and analysis of different formulations. The chosen formulation is assessed on a large-scale building example from the OptiControl project and shown to outperform current control practice.

\section{A. Dealing With Uncertainty}

In the presence of uncertainties, it is generally preferable instead of finding the optimal open-loop input sequence to optimize over closed-loop policies, i.e., to assume that the predicted control inputs at each time step are a state feedback controller (or, equivalently, a disturbance feedback controller). Hence the predicted control input at time $t$ is formulated as a function of the states from now up to time $t$, or, equivalently, as a function of the disturbances that have happened from now up to time $t-1$.

Finding optimal closed-loop policies involves the optimization over an infinite-dimensional function space and is not tractable except for very special cases. It is therefore a common procedure to restrict the optimization to a finite dimensional subspace of the policies, i.e., to choose a control parameterization and optimize over its parameters.

A popular approach is to use a fixed feedback gain [13]-[15], where the control inputs $u$ are parameterized as $u=$ $K x+c$, with $K$ being some stabilizing linear feedback control laws and the perturbation sequence $c$ being the optimization variable. A natural improvement is to simultaneously optimize over both the feedback gain and the perturbation sequence. However, in general, with this approach, the predicted state and input sequences are nonlinear functions of the sequence of state feedback gains and hence it results in a nonconvex set of feasible decision variables.

Inspired from the results in optimization theory on robust optimization problems, in particular, on 
so-called adjustable robust counterpart [16] problems, Löfberg [17] and van Hessem and Bosgra [18] propose to parameterize the control inputs to be affine functions of the disturbances, which leads to a convex set of feasible decision variables. This affine disturbance feedback parameterization is shown to be equivalent to the affine state feedback parameterization in [19]. A more general approach is that of nonlinear disturbance feedback, where the decision variables are the coefficients of a linear combination of nonlinear basis functions of the disturbance [20]. In this work, it is investigated how the use of Affine Disturbance Feedback can be extended to the stochastic setting.

\section{B. Formulation of Chance Constraints}

For problems, where it is not necessary to apply hard constraints, the use of chance constraints can be beneficial because it allows to formulate explicitly the tradeoff between performance and constraint satisfaction [21]. Let $x \in \mathbb{R}^{n_{x}}$ be the system state, $w \in \mathbb{R}^{n_{w}}$ the disturbance, and $\xi \in \mathbb{R}^{r \times n_{x}}$, $\delta \in \mathbb{R}^{r \times n_{w}}$, as well as $\varepsilon \in \mathbb{R}^{r}$ be constants. Chance constraints are assumed to be of the form

$$
\mathbb{P}\left[\xi_{i} x+\delta_{i} w \leq \varepsilon_{i}\right] \geq 1-\beta_{i}, \quad i=1, \ldots, r
$$

where $\beta_{i} \in(0,1)$ is the probability of constraint violation. Chance constraints were first introduced by [22] and have been studied extensively in the field of stochastic programming [23]-[25]. The standard approach in stochastic programming is to consider discrete distributions and then to look at different scenarios, which can be computationally very demanding. Various approaches have been proposed for stochastic MPC, see [26]-[28] and the references therein.

\section{Notation}

The real number set is denoted by $\mathbb{R}$, the set of nonnegative integers by $\mathbb{N}\left(\mathbb{N}_{+}:=\mathbb{N} \backslash\{0\}\right)$, the set of consecutive nonnegative integers $\{j, \ldots, k\}$ by $\mathbb{N}_{j}^{k}$. Denote by $\mathbf{I}_{n} \in\{0,1\}^{n \times n}$ the identity matrix, by $\mathbf{0}_{n \times m} \in\{0\}^{n \times m}$ the zero matrix and by $\mathbf{0}$ without subscript the zero matrix with dimension deemed obvious by context. $\otimes$ denotes the Kronecker product. Let $\operatorname{vec}(x, y):=\left[x^{T} y^{T}\right]^{T}$ denote the vertical concatenation of vectors $x$ and $y$ and let $\operatorname{vec}(A)$ be the vertical concatenation of the columns of matrix $A$, i.e., if $A=\left[a_{1}, \ldots, a_{n}\right]$, then $\operatorname{vec}(A)=\operatorname{vec}\left(a_{1}, \ldots, a_{n}\right)$.

\section{Problem Setting}

Consider the following discrete-time LTI system:

$$
x^{+}=A x+B u+E w
$$

with system state $x \in \mathbb{R}^{n_{x}}$, control input $u \in \mathbb{R}^{n_{u}}$, and stochastic disturbance $w \in \mathbb{R}^{n_{w}}$.

Assumption 1: $(A, B)$ is stabilizable and at each sample instant a measurement of the state is available.

Assumption 2: The disturbance is assumed to be independent and identically distributed (i.i.d.) and to follow a multivariate normal distribution, $w \sim \mathcal{N}\left(\mathbf{0}_{n_{w} \times 1}, \mathbf{I}_{n_{w}}\right)$.
In the following, predictions about the system's evolution over a finite planning horizon are used to define suitable control policies. Consider the prediction horizon $N \in \mathbb{N}_{+}$and define

$$
\begin{aligned}
\mathbf{x} & :=\left[x_{0}^{T} \ldots x_{N}^{T}\right]^{T} \in \mathbb{R}^{(N+1) n_{x}} \\
\mathbf{u} & :=\left[u_{0}^{T} \ldots u_{N-1}^{T}\right]^{T} \in \mathbb{R}^{N n_{u}} \\
\mathbf{w} & :=\left[w_{0}^{T} \ldots w_{N-1}^{T}\right]^{T} \in \mathbb{R}^{N n_{w}}
\end{aligned}
$$

where $x_{0}$ denotes the current measured value of the state and $x_{k+1}:=A x_{k}+B u_{k}+E w_{k}, k \in \mathbb{N}_{0}^{N-1}$ denotes the predictions of the state after $k$ time instants into the future. Let furthermore prediction dynamics matrices $\mathbf{A}, \mathbf{B}$, and $\mathbf{E}$ be such that

$$
\mathbf{x}=\mathbf{A} x_{0}+\mathbf{B u}+\mathbf{E w} \text {. }
$$

Polytopic constraints on inputs $\mathbf{u}$ and chance constraints on states $\mathbf{x}$ over the prediction horizon $N$ are given as

$$
\begin{aligned}
\mathbf{S u} & \leq \mathbf{s} \\
\mathbb{P}\left[\mathbf{G}_{j} \mathbf{x} \leq \mathbf{g}_{j}\right] & \geq 1-\alpha_{\mathbf{x}, j \quad} \quad \forall j \in \mathbb{N}_{1}^{r(N+1)}
\end{aligned}
$$

where $\mathbf{S} \in \mathbb{R}^{q N \times n_{u} N}, \mathbf{s} \in \mathbb{R}^{q N}, \mathbf{G} \in \mathbb{R}^{r(N+1) \times n_{x}(N+1)}, \mathbf{g} \in$ $\mathbb{R}^{r(N+1)}$, and $\alpha_{\mathbf{x}, j} \in(0,0.5]$ denotes the probability level of constraint violation for row $j$ of the constraints on $\mathbf{x}$.

Remark 1: $\alpha_{\mathbf{x}, j}$ is restricted to be in $(0,0.5]$ as it can then be reformulated into a second order cone (SOC) constraint.

$x_{0}$ is measured, however, the predicted states $x_{1}, \ldots, x_{N}$ are uncertain. To address this uncertainty in the MPC formulation, consider for predicted control inputs $u_{1}, \ldots, u_{N}$ a causal state feedback controller, or equivalently, a causal disturbance feedback controller

$$
u_{k}=\phi_{k}\left(x_{1}, \ldots, x_{k}\right)=\mu_{k}\left(w_{0}, \ldots, w_{k-1}\right) .
$$

Remark 2: The state feedback controller is called causal as it strictly depends on the states that have already been realized. It is equivalent to consider a disturbance feedback controller, as the disturbances can be straightforwardly computed with given states and known inputs.

The aim is to solve the following stochastic finite horizon optimal control problem (SFHOCP).

Problem 1 (SFHOCP):

$$
\begin{aligned}
\min _{\mathbf{u}} \mathbb{E} & {\left[\sum_{k=0}^{N-1} l_{k}\left(x_{k}, u_{k}\right)+l_{N}\left(x_{N}\right)\right] } \\
\text { s.t. } & \mathbf{S u} \leq \mathbf{s} \\
& \mathbb{P}\left[\mathbf{G}_{j} \mathbf{x} \leq \mathbf{g}_{j}\right] \geq 1-\alpha_{\mathbf{x}, j} \quad \forall j \in \mathbb{N}_{1}^{r(N+1)} \\
& \mathbf{x}=\mathbf{A} x_{0}+\mathbf{B u}+\mathbf{E w} \\
& \mathbf{w} \sim \mathcal{N}\left(\mathbf{0}_{n_{w} N \times 1}, \mathbf{I}_{n_{w} N}\right) \\
& u_{k}=\mu_{k}\left(w_{0}, \ldots, w_{k-1}\right)
\end{aligned}
$$

for some stage cost $l_{k}\left(x_{k}, u_{k}\right)$ and some terminal cost $l_{N}\left(x_{N}\right)$. Note that in Problem I, the aim is not to find an optimal control input sequence, but to find an optimal control policy as defined in (7) taking into account that there is recourse/ feedback at every future stage/time step. This SFHOCP cannot be readily solved, as it involves the optimization over an infinitedimensional function space. This problem is tackled in this 
brief paper by restricting the policies to a finite-dimensional subspace. Furthermore, the above problem involves chance constraints that are to be reformulated so that they can be efficiently handled in the optimization problem. Sections III and IV deal with approximations and reformulations to turn Problem I into a tractable MPC problem. First, Affine Disturbance Feedback is introduced and then, three reformulations of the chance constraints are considered.

\section{AfFine Disturbance FeEdBACK FOR STOCHASTIC MPC}

Affine Disturbance Feedback (ADF) has been successfully used in robust control [16]-[19].

Definition 1 [Affine Disturbance Feedback]: Define $\mu_{k}$ : $\mathbb{R}^{n_{w} N} \rightarrow \mathbb{R}^{n_{u}}$

$$
\mu_{k}(\mathbf{w}):=\sum_{j=0}^{k-1} M_{k, j} w_{j}+h_{k}, \quad k \in \mathbb{N}_{0}^{N-1}
$$

with $M_{k, j} \in \mathbb{R}^{n_{u} \times n_{w}}$ and $h_{k} \in \mathbb{R}^{n_{u}}$.

Let $\mu(\mathbf{w}):=\left[\begin{array}{llll}u_{0}^{T} & \mu_{1}^{T}(\mathbf{w}) & \ldots & \mu_{N-1}^{T}(\mathbf{w})\end{array}\right]^{T}$. We can write $\mu(\mathbf{w})=\mathbf{M w}+\mathbf{h}$ defining

$$
\mathbf{M}:=\left[\begin{array}{cccc}
0 & \cdots & \cdots & 0 \\
M_{1,0} & 0 & \ddots & 0 \\
\vdots & \ddots & \ddots & \vdots \\
M_{N-1,0} & \cdots & M_{N-1, N-2} & 0
\end{array}\right], \mathbf{h}:=\left[\begin{array}{c}
h_{0} \\
\vdots \\
\vdots \\
h_{N-1}
\end{array}\right] .
$$

Remark 3: Note that because of the structure of $\mathbf{M}$ for the computation of the control input at time step $k$, only the disturbances up to time $k-1$ are taken into account. Note also, that the very first control input is not a function of the disturbance, but $u_{0}=h_{0}$.

Applying such a control policy is also referred to as closedloop prediction MPC. In the ADF MPC problem we choose to minimize a quadratic cost function.

Definition 2 [Cost function]:

$$
J(\mathbf{x}, \mathbf{M}, \mathbf{h}):=\mathbb{E}\left[\mathbf{x}^{T} \mathbf{Q} \mathbf{x}+(\mathbf{M w}+\mathbf{h})^{T} \mathbf{R}(\mathbf{M w}+\mathbf{h})\right]
$$

where $\mathbf{Q}=\mathbf{Q}^{T} \succeq 0$ and $\mathbf{R}=\mathbf{R}^{T} \succ 0$. With $\mathbb{E}[\mathbf{w}]=\mathbf{0}$ and $\mathbb{E}\left[\mathbf{w} \mathbf{w}^{T}\right]=\mathbf{I}$ and using (4), (7), and (8), the cost function is given as $J_{N}: \mathbb{R}^{n_{x}} \times \mathbb{R}^{n_{u} N \times n_{w} N} \times \mathbb{R}^{n_{u} N} \rightarrow \mathbb{R}$

$$
\begin{aligned}
& J_{N}\left(x_{0}, \mathbf{M}, \mathbf{h}\right)=x_{0}^{T} \mathbf{A}^{T} \mathbf{Q A} x_{0}+\operatorname{Tr}\left[\mathbf{E}^{T} \mathbf{Q E}\right]+\cdots \\
& \mathbf{h}^{T}\left(\mathbf{B}^{T} \mathbf{Q B}+\mathbf{R}\right) \mathbf{h}+2 \mathbf{h}^{T} \mathbf{B}^{T} \mathbf{Q A} x_{0}+\cdots \\
& \operatorname{Tr}\left[\mathbf{M}^{T} \mathbf{B}^{T} \mathbf{Q B M}+\mathbf{M}^{T} \mathbf{R} \mathbf{M}+2 \mathbf{M}^{T} \mathbf{B}^{T} \mathbf{Q E}\right] .
\end{aligned}
$$

Proposition 1: The cost function in (10) is a convex quadratic function of the decision variables $\mathbf{M}$ and $\mathbf{h}$.

Proof: Convexity of the quadratic cost function is preserved because of the policies being affine and under expectation.

Using affine disturbance feedback, an approximation of the SFHOCP in Problem II can be stated.

Problem 2 (Approximate SFHOCP Using ADF):

$$
\left(\mathbf{M}^{*}\left(x_{0}\right), \mathbf{h}^{*}\left(x_{0}\right)\right):=\arg \min _{(\mathbf{M}, \mathbf{h}) \in \Pi\left(x_{0}\right)} J_{N}\left(x_{0}, \mathbf{M}, \mathbf{h}\right) .
$$

For state $x_{0}$, the set of admissible $\operatorname{ADF}$ policies $(\mathbf{M}, \mathbf{h})$ is given by the set

$$
\Pi\left(x_{0}\right):=\left\{\begin{array}{l|l}
(\mathbf{M}, \mathbf{h}) & \begin{array}{l}
(\mathbf{M}, \mathbf{h}) \text { satisfies }(9) \\
\mathbb{P}\left[\mathbf{S}_{i}(\mathbf{M w}+\mathbf{h}) \leq \mathbf{s}_{i}\right] \geq 1-\alpha_{\mathbf{u}, i} \\
\mathbb{P}\left[\mathbf{G}_{j}\left(\mathbf{A} x_{0}+\mathbf{B h}+\mathbf{B M w}+\mathbf{E w}\right) \leq g_{j}\right] \\
\geq 1-\alpha_{\mathbf{x}, j} \quad \forall i \in \mathbb{N}_{1}^{q N}, \forall j \in \mathbb{N}_{1}^{r(N+1)} \\
\mathbf{w} \sim \mathcal{N}\left(\mathbf{0}_{n_{w} N \times 1}, \mathbf{I}_{n_{w} N}\right)
\end{array}
\end{array}\right\} .
$$

Following the standard MPC procedure, the optimal control input $u^{*}\left(x_{0}\right)$ is then given by $u^{*}(x)=h_{0}^{*}(x)$ and the closedloop trajectory evolves according to $x^{+}=A x+B u^{*}(x)+E w$.

Note that the disturbances have an infinite support and the inputs are, except for the very first input, a function of the disturbances (see Remark 3). Therefore it is only possible to define a hard constraint on the first input (see also Remark 4). Remark 4: Hard constraints on the control inputs are approximated with small values of $\alpha_{\mathbf{u}, i}$ to fulfill the hard constraints with a high probability. Very small values, however, can lead to infeasibility. Note that the input constraints are not violated when this strategy is applied in closed-loop because of the structure of $\mathbf{M}$, i.e., on the first predicted state, which is applied, a hard constraint is imposed.

\section{Chance Constraint Reformulations}

All chance constraint reformulations are shown for the chance constraints on the states. They can be applied to the chance constraints on the inputs accordingly, which is omitted for brevity.

\section{A. ADF with Quantile Function}

Because the constraints in Problem 2 are bi-affine in the decision variables and disturbances and the disturbances are normally distributed, the individual chance constraints can be equivalently formulated as deterministic SOC constraints [23] as

$$
\begin{aligned}
& \mathbb{P}\left[\mathbf{G}_{j}\left(\mathbf{A} x_{0}+\mathbf{B h}+\mathbf{B M w}+\mathbf{E w}\right)-\mathbf{g}_{j} \leq 0\right] \\
& \quad \geq 1-\alpha_{\mathbf{x}, j} \\
& \Leftrightarrow \mathbf{G}_{j}\left(\mathbf{A} x_{0}+\mathbf{B h}\right) \\
& \quad \leq \mathbf{g}_{j}-\Phi^{-1}\left(1-\alpha_{\mathbf{x}, j}\right)\left\|\mathbf{G}_{j}(\mathbf{B M}+\mathbf{E})\right\|_{2}
\end{aligned}
$$

where $\Phi$ is the standard Gaussian cumulative distribution function and its inverse the Quantile Function (QF). The inequalities (12) are SOC constraints that are convex in the decision variables $\mathbf{M}$ and $\mathbf{h}$.

Problem 3 (ADF With $Q F)$ :

$$
\left(\mathbf{M}^{*}\left(x_{0}\right), \mathbf{h}^{*}\left(x_{0}\right)\right):=\arg \min _{(\mathbf{M}, \mathbf{h}) \in \Pi_{Q F}\left(x_{0}\right)} J_{N}\left(x_{0}, \mathbf{M}, \mathbf{h}\right) .
$$

For state $x_{0}$, the set of admissible ADF policies $(\mathbf{M}, \mathbf{h})$ when using QFs is

$$
\Pi_{\mathrm{QF}}\left(x_{0}\right):=\left\{\begin{array}{l|l}
(\mathbf{M}, \mathbf{h}) & \begin{array}{l}
(\mathbf{M}, \mathbf{h}) \text { satisfies }(8) \\
\mathbf{S}_{i} \mathbf{h} \leq \mathbf{s}_{i}-\Phi^{-1}\left(1-\alpha_{\mathbf{u}, i}\right)\left\|\mathbf{S}_{i} \mathbf{M}\right\|_{2} \\
\mathbf{G}_{j}\left(\mathbf{A} x_{0}+\mathbf{B h}\right) \leq \mathbf{g}_{j} \\
-\Phi^{-1}\left(1-\alpha_{\mathbf{x}, j}\right)\left\|\mathbf{G}_{i}(\mathbf{B M}+\mathbf{E})\right\|_{2} \\
\forall i \in \mathbb{N}_{1}^{q N} \quad \forall j \in \mathbb{N}_{1}^{r(N+1)}
\end{array}
\end{array}\right\},
$$


Problem 3 is equivalent to Problem 2. Problem 3 is a convex problem involving SOC constraints.

\section{B. ADF with Bounds on the Disturbance Set}

The reformulation of the chance constraints in Section III led to an SOC problem, this can however be time-consuming to compute for large-scale systems. Therefore, this section deals with a linear approximation.

We propose to replace the chance constraint in (11) with the following robust constraint:

$$
\begin{aligned}
& \mathbf{G}_{j}\left(\mathbf{A} x_{0}+\mathbf{B h}+\mathbf{B M} \mathbf{M}+\mathbf{E w}\right)-\mathbf{g}_{j} \leq 0 \quad \forall w \in \mathcal{W} \\
& \mathcal{W}:=\left\{w \in \mathbb{R}^{n_{w}} \mid\|w\|_{\infty} \leq \Omega_{\mathbf{x}, j}\right\}
\end{aligned}
$$

where $\Omega_{\mathbf{x}, j}$ is chosen according to the following Theorem 1, which is directly derived by some algebraic reformulations of (11) and use of [21, Th. 2b]. See Appendix I.

\section{Theorem 1 (Probability Bound):}

For $\mathbf{w} \sim \mathcal{N}(\mathbf{0}, \mathbf{I})$ and for $\Omega_{\mathbf{x}, j}>1$ the following probability bound of infeasibility holds:

$$
\begin{aligned}
\mathbb{P}\left[\mathbf { G } _ { j } \left(\mathbf{A} x_{0}+\mathbf{B h}+\mathbf{B M w}\right.\right. & \left.+\mathbf{E w})-\mathbf{g}_{j}>0\right] \\
& \leq \sqrt{e} \cdot \Omega_{\mathbf{x}, j} \cdot \exp \left(-\frac{\Omega_{\mathbf{x}, j}^{2}}{2}\right) .
\end{aligned}
$$

Proof: See Appendix I.

This theorem provides the following performance guarantee.

Corollary 1: If $\Omega_{\mathbf{x}, j}$ is chosen according to Theorem 1, i.e., such that $\alpha_{\mathbf{x}, j} \geq \sqrt{e} \cdot \Omega_{\mathbf{x}, j} \cdot \exp \left(-\Omega_{\mathbf{x}, j}^{2} / 2\right)$, and (13) is applied, then the chance constraint in (11) is satisfied.

As a result, this approximation with Bounds on the Disturbance Set (BDS) can be used to reformulate Problem 1 as a tractable robust optimization problem.

Problem 4 (ADF With BDS):

$$
\left(\mathbf{M}^{*}\left(x_{0}\right), \mathbf{h}^{*}\left(x_{0}\right)\right):=\arg \min _{(\mathbf{M}, \mathbf{h}) \in \Pi_{B D S}\left(x_{0}\right)} J_{N}\left(x_{0}, \mathbf{M}, \mathbf{h}\right) .
$$

For state $x_{0}$, the set of admissible $\operatorname{ADF}$ policies $(\mathbf{M}, \mathbf{h})$ with $\mathrm{BDS}$ is given by

$$
\Pi_{\mathrm{BDS}}\left(x_{0}\right):=\left\{\begin{array}{l|l}
(\mathbf{M}, \mathbf{h}) & \begin{array}{l}
(\mathbf{M}, \mathbf{h}) \text { satisfies }(8) \\
\mathbf{S}_{\mathbf{i}} \mathbf{h} \leq \mathbf{S}_{\mathbf{i}}-\max _{\|w\|_{\infty} \leq \mathbf{\Omega}_{\mathbf{u}, i}} \mathbf{S}_{\mathbf{i}} \mathbf{M w} \\
\mathbf{G}_{\mathbf{j}}\left(\mathbf{A} x_{0}+\mathbf{B h}\right) \leq \mathbf{g}_{\mathbf{j}} \\
-\max _{\|w\|_{\infty} \leq \Omega_{\mathbf{x}, j} \mathbf{G}_{\mathbf{j}}(\mathbf{B M w}+\mathbf{E w})} \\
\forall i \in \mathbb{N}_{1}^{q N} \quad \forall j \in \mathbb{N}_{1}^{r(N+1)}
\end{array}
\end{array}\right\} .
$$

Problem 4 can be solved as a standard QP, see [12].

Theorem 2 ( $A D F$ With BDS): For Problem 4, the following statements hold: (1) Problem 4 is a conservative approximation of Problem 2 in the sense that the level of constraint violation is strictly smaller than $\alpha$.

(2) Problem 4 is convex:

Proof: 1) The statement follows trivially from Theorem 1 and Corollary 1. It can also be seen from the fact that linear constraints are used for inner approximations of SOC constraints.

2) $\Pi_{\mathrm{BDS}}\left(x_{0}\right)$ is convex (see [12]). This together with the cost function of Problem 4 being convex according to Proposition 1 establishes the assertion.

\section{Approximation with Fixed Feedback}

Problem 3 uses an equivalent deterministic reformulation of the chance constraints, but leads to a SOC problem, whereas Problem 4 leads to linear constraints, but uses an approximation of the chance constraints. These advantages are combined with a third formulation, Fixed Feedback (FF). We propose to optimize over the amount of feedback that is considered, i.e., to optimize over a scaling factor that multiplies an a priori determined fixed feedback matrix.

The feedback is computed by taking the average over some number $n_{\mathbf{M}}$ of optimal feedback matrices $\mathbf{M}_{t}^{*}(x)$ that are obtained by solving the SOC problem at time $t$ for the corresponding measured state $x$

$$
\overline{\mathbf{M}}:=\frac{1}{n_{\mathbf{M}}} \sum_{t=1}^{n_{\mathbf{M}}} \mathbf{M}_{t}^{*}(x) .
$$

This means that for some number of steps, the full SOC problem is solved (this could be done in a real implementation in simulation only) and the resulting feedback matrices are stored. Then the average of these matrices is computed. For all subsequent simulations, the feedback matrix is fixed to the average matrix and the optimization is restricted to the scaling factor.

Problem 5 (ADF With FF):

$$
\left(\gamma^{*}\left(x_{0}\right), \mathbf{h}^{*}\left(x_{0}\right)\right):=\arg \min _{(\gamma \overline{\mathbf{M}}, \mathbf{h}) \in \Pi_{F F}\left(x_{0}\right)} J_{N}\left(x_{0}, \gamma \overline{\mathbf{M}}, \mathbf{h}\right) .
$$

For state $x_{0}$, the set of admissible ADF policies $(\mathbf{M}, \mathbf{h})$ when using FF is given by

$$
\Pi_{\mathrm{FF}}\left(x_{0}\right):=\left\{\begin{array}{l|l}
(\gamma \overline{\mathbf{M}}, \mathbf{h}) & \begin{array}{l}
\mathbf{S}_{i} \mathbf{h} \leq \mathbf{s}_{i}-\Gamma_{i}\left\|\mathbf{S}_{i} \gamma \overline{\mathbf{M}}\right\|_{2} \\
\mathbf{G}_{j}\left(\mathbf{A} x_{0}+\mathbf{B h}\right) \leq \mathbf{g}_{j} \\
-\Upsilon_{j}\left\|\mathbf{G}_{i}(\mathbf{B} \gamma \overline{\mathbf{M}}+\mathbf{E})\right\|_{2} \\
\gamma \in[0,1] \\
\forall i \in \mathbb{N}_{1}^{q N} \quad \forall j \in \mathbb{N}_{1}^{r(N+1)}
\end{array}
\end{array}\right\}
$$

where $\Gamma_{i}:=\Phi^{-1}\left(1-\alpha_{\mathbf{u}, i}\right)$ and $\Upsilon_{j}:=\Phi^{-1}\left(1-\alpha_{\mathbf{x}, j}\right)$ and $\gamma \in[0,1]$ is used to optimize how much feedback is taken into account ranging from none (i.e., open-loop prediction, $\mathbf{M}=0$ ) to the full preoptimized feedback $\overline{\mathbf{M}}$.

\section{SMALL-SCALE EXAMPLE}

To test the proposed strategies, a simplified version of the building example in [29] is examined. This example on one hand reflects basic properties of the (more complicated) building example to be tackled, so it can serve as an example for comparison and selection of the proposed algorithm, but it is simple enough to carry out many simulations and understand the basic behavior. The system dynamics have the form of (2), but with an external input $v \in \mathbb{R}^{n_{v}}$ and matrix $V$ of appropriate size to account for the weather forecast

$$
x_{k+1}=A x_{k}+B u_{k}+V v_{k}+E w_{k} .
$$

Let $x_{k}=\left[\begin{array}{lll}x_{(1)} & x_{(2)} & x_{(3)}\end{array}\right]^{T}$ denote the state, where $x_{(1)}$ is the room temperature, $x_{(2)}$ the temperature in the wall connected with another room, and $x_{(3)}$ the temperature in the 
wall connected to the outside. There is a prediction for the external input $v_{k}=\left[\begin{array}{lll}v_{(1)} & v_{(2)} & v_{(3)}\end{array}\right]^{T}$, the outside temperature $v_{(1)}$, the solar radiation $v_{(2)}$, and the internal heat gains $v_{(3)}$ (people, appliances). The predictions of internal gains are assumed to be perfect in this example, i.e., the realization is equal to the prediction. However, for the weather variables, the realization is equal to the prediction plus some random noise $w_{(1)}$ and $w_{(2)}$, which is assumed to consist of i.i.d. Gaussian random variables. The control objective is to keep the room temperature $>21{ }^{\circ} \mathrm{C}$ with minimum energy. The single available input $u_{(1)}$ is the heating, which is constrained to $0 \leq u_{(1)} \leq 45\left[\mathrm{~W} / \mathrm{m}^{2}\right]$. The time step is $1 \mathrm{~h}$. The system matrices are given as

$$
\begin{aligned}
& A=\left[\begin{array}{lll}
0.8511 & 0.0541 & 0.0707 \\
0.1293 & 0.8635 & 0.0055 \\
0.0989 & 0.0032 & 0.7541
\end{array}\right] \quad B=\left[\begin{array}{l}
0.070 \\
0.006 \\
0.004
\end{array}\right] \\
& V=\left[\begin{array}{lll}
0.02221 & 0.00018 & 0.0035 \\
0.00153 & 0.00007 & 0.0003 \\
0.10318 & 0.00001 & 0.0002
\end{array}\right] \quad E=\left[\begin{array}{ll}
0.4 & 0.014 \\
0.028 & 0.006 \\
1.857 & 0.001
\end{array}\right] .
\end{aligned}
$$

The following five control strategies were compared.

1) CLP-BDS: Closed-loop prediction MPC with BDS (Problem 4);

2) OLP-BDS: Open-loop prediction MPC with BDS (Problem 4, with $\mathbf{M}=0$ );

3) CLP-QF: Closed-loop prediction MPC using the QF (Problem 3);

4) OLP-QF: Open-loop prediction MPC using the QF (Problem 3, with $\mathbf{M}=0$ );

5) FF-QF: Fixed feedback using the QF (Problem 5).

To obtain an upper bound on the performance, the feedback matrix is computed by first computing the feedback matrices for the whole simulation and determining their average and then starting the simulation with the precomputed feedback matrix.

Compared to (2), we have in this example an external input, i.e., a time-varying linear system. To compare the above strategies, we first consider a constant external input to focus on the effects of the formulation. We assume that the random noise takes its average value and we observe how far away from the nominal constraint the strategy takes us, i.e., for how much backup from the nominal constraint the controller is planning because of the uncertainty (Investigation 1). Second, we take into account the variation in external input and observe how far away from the nominal constraint we are in presence of the external variations (Investigation 2). Third, the external input is held constant, the random noise is varied, and a Monte Carlo study is carried out to evaluate how often the constraints are violated (Investigation 3).

Investigation 1: The external input prediction was kept constant at $v_{1}=10.6{ }^{\circ} \mathrm{C}, v_{2}=18 \mathrm{~W} / \mathrm{m}^{2}$, and $v_{3}=18 \mathrm{~W} / \mathrm{m}^{2}$. The random noise was set to its mean value, $w_{1}=w_{2}=0$, i.e., the external input realization was equal to the prediction, and $\alpha_{\mathbf{u}, i}=0.0003$ to have a high probability to fulfill the (hard) input constraints, and $\alpha_{\mathbf{x}, j}$ was varied.
Investigation 2: The external input prediction was assumed to be time-varying. The random noise was set to its mean value, $w_{1}=w_{2}=0$, i.e., the external input realization was equal to the prediction, $\alpha_{\mathbf{u}, i}=0.0003$, and $\alpha_{\mathbf{x}, j}$ was varied.

Investigation 3: The external input prediction was kept constant at $v_{1}=10.6{ }^{\circ} \mathrm{C}, v_{2}=18 \mathrm{~W} / \mathrm{m}^{2}$, and $v_{3}=$ $18 \mathrm{~W} / \mathrm{m}^{2}$. The random noise following a standard Gaussian distribution was applied to the system in a Monte Carlo study with 70 samples, i.e., the external input realization differed from the prediction. $\alpha_{\mathbf{u}, i}=0.0003$, and $\alpha_{\mathbf{x}, j}$ was varied.

All investigations were carried out for three days $=72 \mathrm{~h}$, with a prediction horizon of $N=6$. To minimize energy only the control inputs were penalized, i.e., the cost function in (10) was used, but with $\mathbf{Q}=\mathbf{0}$

$$
J_{N}^{\mathrm{Ex} 1}\left(x_{0}, \mathbf{M}, \mathbf{h}\right):=\mathbf{h}^{T} \mathbf{R h}+\operatorname{Tr}\left[\mathbf{M}^{T} \mathbf{R M}\right] .
$$

\section{A. Results}

Investigation 1: In Fig. 1, the control performance in terms of energy use of the five investigated strategies for different levels of $\alpha_{\mathbf{x}, j}$ is depicted. OLP-BDS has highest energy usage. Considering instead the closed-loop prediction formulation (CLP-BDS) improves the performance clearly. OLP-QF uses even less energy, and again, using the closed-loop formulation further improves the performance (CLP-QF). To summarize, closed-loop prediction improves the performance and using $\mathrm{QF}$ is better than BDS. The performance of CLP-QF and FF-QF is almost identical. Fig. 3 shows the room temperature profile of the same investigation. As expected, OLP-BDS is very conservative and CLP-QF is the least conservative. Fig. 5 depicts the corresponding heating input that was applied. Note that if the prediction horizon length $N$ is increased, then it is not possible anymore to get a feasible solution for OLP-BDS.

Investigation 2: In Fig. 2, the control performance in terms of energy use of the five investigated strategies for different levels of $\alpha_{\mathbf{x}, j}$ is depicted. The results are very similar to the one of Investigation 1, i.e., QF is superior to BDS and CLP is better than OLP. The applied energy is less in this investigation as additional heat gains were introduced because of the outside temperature, solar radiation, and internal gains. Figs. 4 and 6 show the room temperature profile and the heating input, respectively. Here, the effect of the varying outside weather conditions can be clearly seen.

Investigation 3: The comparison of the control performance in terms of energy use versus $\alpha_{\mathbf{x}, j}$ is similar to the results from the first two investigations. QF is better than BDS and the closed-loop controllers outperform the open-loop controllers (Fig. 7). The tradeoff between energy use and allowed constraint violation level can clearly be seen for all strategies.

From the results, it can be concluded that QF can be expected to outperform BDS, whereas with the FF-QF formulation, almost a similar performance as CLP-QF can be achieved. Furthermore, the parameter $\alpha$ can be used as a tuning parameter to change the resulting amount of violations. Therefore, the FF-QF was further tested and analyzed on a large-scale building example. 


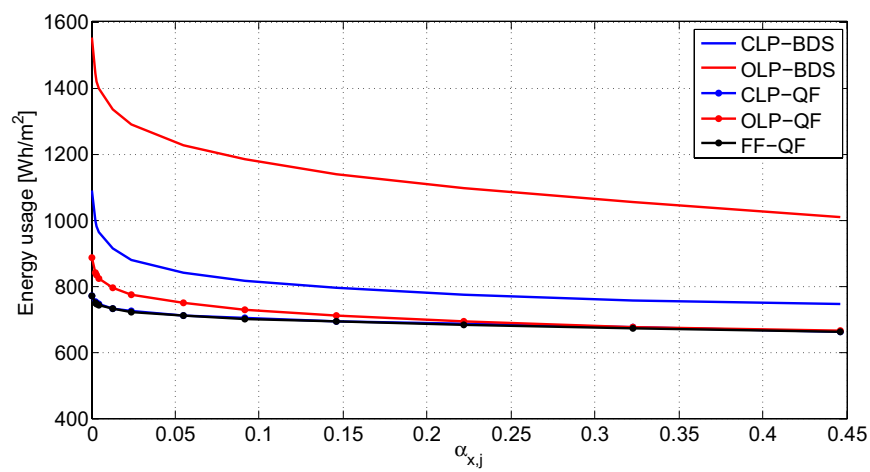

Fig. 1. Investigation 1: energy use $\left[\mathrm{Wh} / \mathrm{m}^{2}\right]$ versus $\alpha_{\mathbf{x}, j}$.

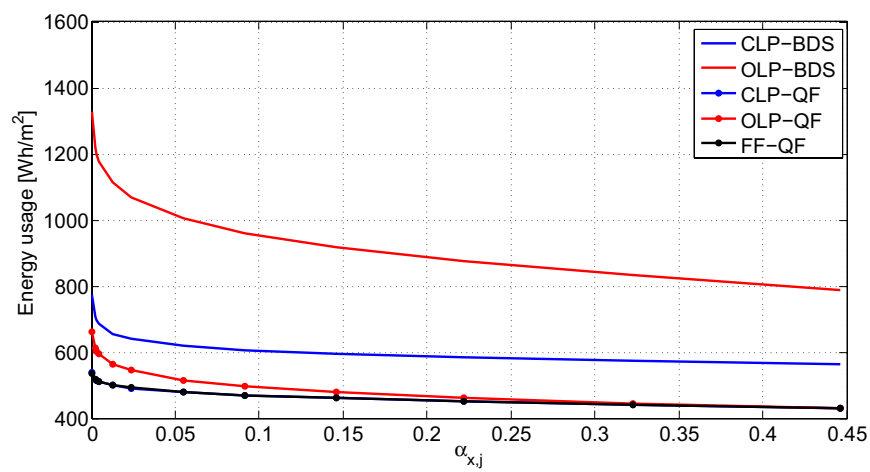

Fig. 2. Investigation 2: energy use $\left[\mathrm{Wh} / \mathrm{m}^{2}\right]$ versus $\alpha_{\mathbf{x}, j}$.

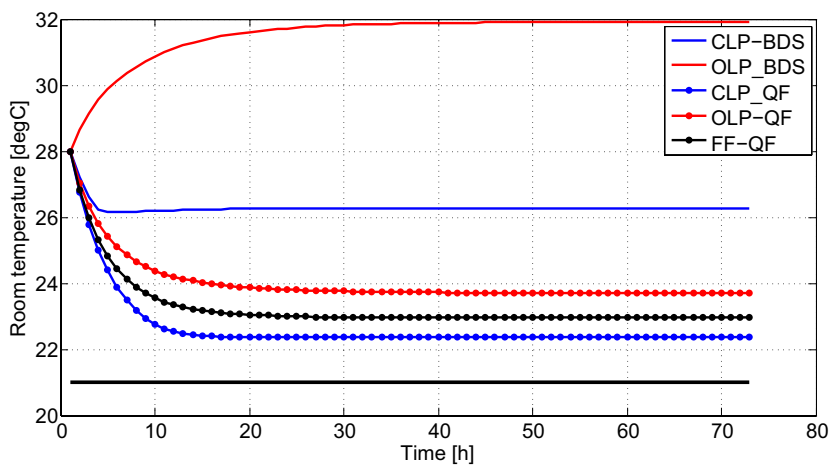

Fig. 3. Investigation 1: room temperature profile [degC] for $\alpha_{\mathbf{x}, j}=0.0003$.

\section{Vi. Example from Building Climate Control}

The stochastic MPC formulation was primarily motivated by the use of building control. To determine the energy savings potential of using stochastic MPC in building control, a largescale simulation study with different buildings and weather conditions was carried out in the framework of the project OptiControl [11]. An excerpt of the results is presented here to highlight the applicability of the proposed method. More details and analyses can be found in [2], [30], and [31].

\section{A. Modeling}

The building dynamics are represented with a bilinear model [2], which is augmented by an autoregressive model of first order driven by Gaussian noise describing the uncertainty in weather predictions. This uncertainty model is obtained from historical weather data [2] and also used in a Kalman filter for filtering the weather predictions. Weather predictions and measurements are obtained from MeteoSwiss [32]. To deal

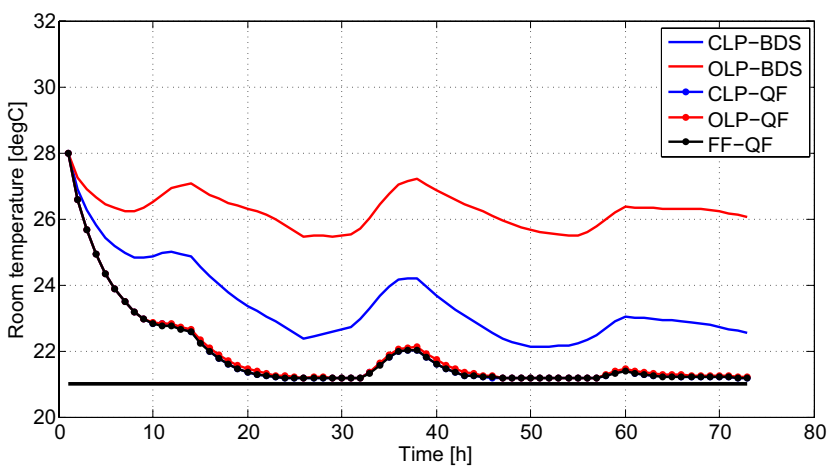

Fig. 4. Investigation 2: room temperature profile [degC] for $\alpha_{\mathbf{x}, j}=0.32$.

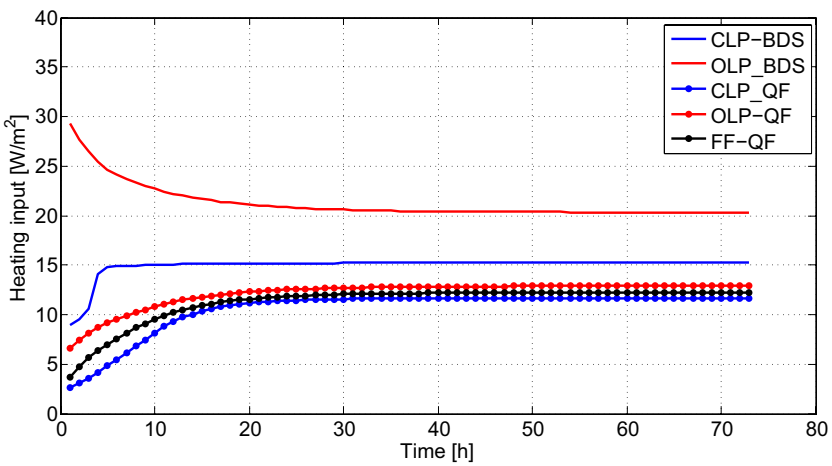

Fig. 5. Investigation 1: heating $\left[\mathrm{W} / \mathrm{m}^{2}\right]$ for $\alpha_{\mathbf{x}, j}=0.0003$.

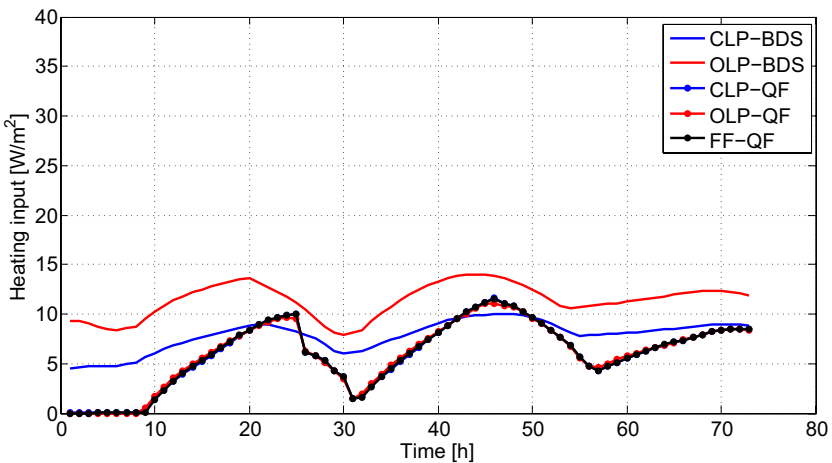

Fig. 6. Investigation 2: heating $\left[\mathrm{W} / \mathrm{m}^{2}\right]$ for $\alpha_{\mathbf{x}, j}=0.32$.

with the bilinear model, we apply a form of sequential linear programming, [31], [33], in which we iteratively linearize around the current solution yielding a time-varying input matrix $B_{u, k}$. At each time step, an MPC problem for the linear system is formulated with the form

$$
x_{k+1}=A x_{k}+B_{u, k} u_{k}+V v_{k}+E w_{k} .
$$

\section{B. Control Strategies and Benchmarks}

Three different control strategies were considered in the investigation as well as a benchmark. All are listed as follows.

Rule-based control: The standard strategy in current practice and used by, amongst others, Siemens Building Technologies is RBC [34]. As the name indicates, RBC determines all control inputs based on a series of rules of the kind if condition then action.

Deterministic MPC: This formulation assumes that the disturbance takes its expected value neglecting the uncertainty. 


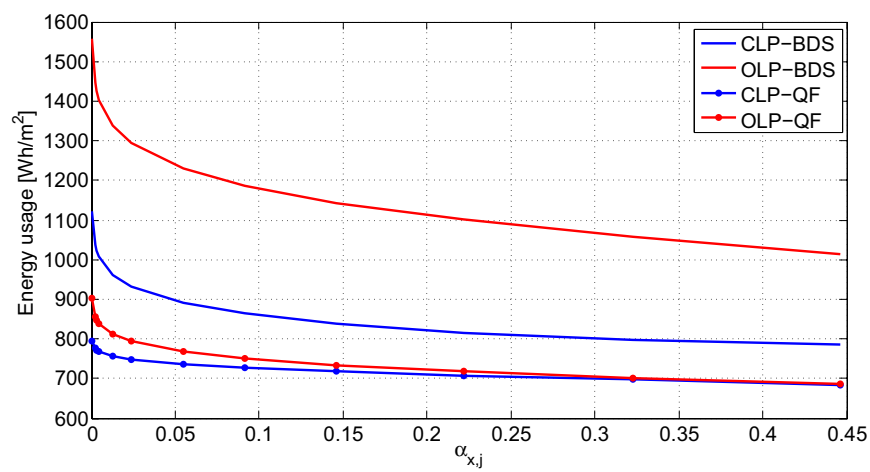

Fig. 7. Investigation 3: average energy use $\left[\mathrm{Wh} / \mathrm{m}^{2}\right]$ from Monte Carlo trials versus $\alpha_{\mathbf{x}, j}$.

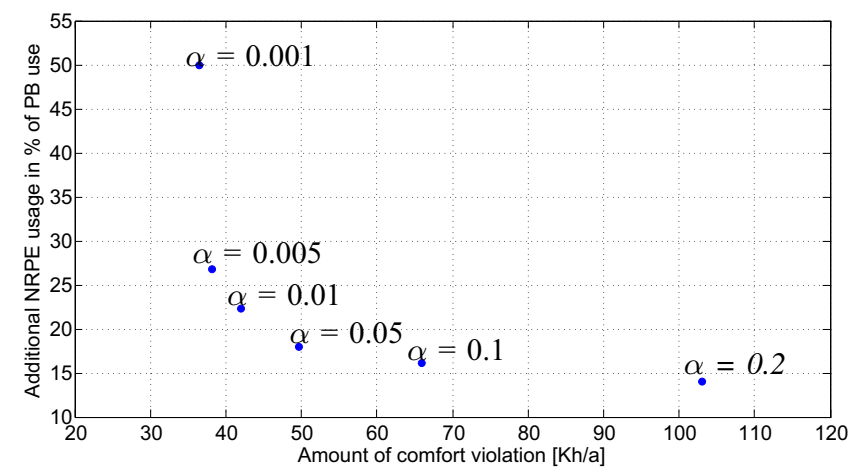

Fig. 8. Tuning of SMPC for Case 1 in [2]. Tradeoff curve of NRPE usage versus comfort violations.

This is equivalent with Problem 1, setting all disturbances equal to zero and assuming hard constraints on the states instead of chance constraints. A prediction horizon of $24 \mathrm{~h}$ and a control horizon of $1 \mathrm{~h}$ was used.

Stochastic MPC: The formulation in Problem 5 with the FF was used with a prediction horizon of $24 \mathrm{~h}$ and a control horizon of $1 \mathrm{~h}$. Deviating from Problem 5, no scaling factor $\gamma$ was used, but the feedback $\overline{\mathbf{M}}$ at each time step was fully applied, and furthermore, soft constraints [35] were used.

Performance bound: PB is defined as optimal control with perfect knowledge of both the system dynamics as well as all future disturbances acting upon the system. PB is not a controller, but a concept that can serve as a benchmark. To compute PB, the same MPC algorithm was used as for DMPC, but with perfect weather predictions.

In this brief paper, the Non-Renewable Primary Energy (NRPE) usage was assessed as well as the amount and number of violations. For this, the cost function in Example 2 is a linear cost function of the form

$$
J_{N}^{\mathrm{Ex} 2}\left(x_{0}, \mathbf{M}, \mathbf{h}\right):=\operatorname{diag}(\mathbf{R})^{T} \mathbf{h} .
$$

According to the standards, a reasonable violation level is $70 \mathrm{Kh} / \mathrm{a}$ (Kelvin hours per annum, $1 \mathrm{Kh}$ denotes the violation by one Kelvin for one hour). The considered buildings and weather scenarios are Cases 1, 2, 3, 7, 17, 18 in [2].

\section{Results}

In Fig. 8, the tuning of Case 1 in [2] is shown by varying the level of constraint violation $\alpha$. A tradeoff curve of NRPE

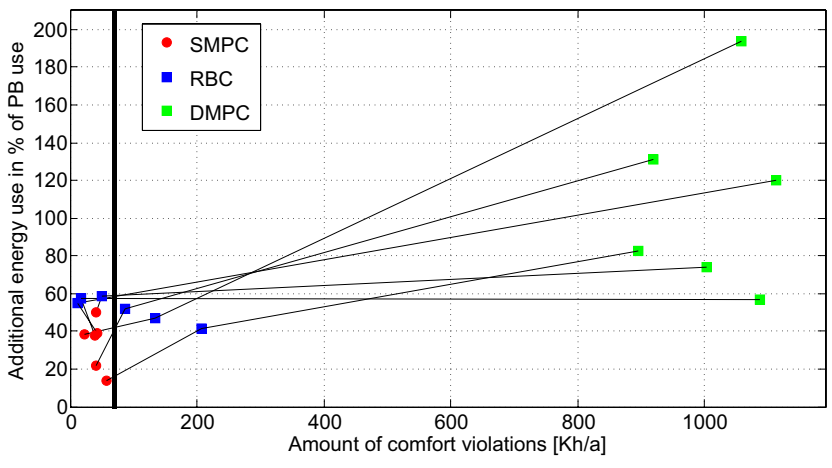

Fig. 9. Comparison of SMPC, RBC, and DMPC for the example cases in [2]. Results of the same case are connected with a line.

usage versus comfort violations is obtained. A comparison of the three control strategies is given in Fig. 9. The NRPE usage is normalized with the NRPE usage of PB and only the additional NRPE usage in \% of PB is considered. The amount of violations is not normalized, as PB has no violations. For each case, the results of the three controllers are connected with a line. The closer to the origin the better, since the origin is equal to the performance of PB. The performance of DMPC (without any tuning) is worst for all cases, as it gives rise to the largest NRPE usage and the largest violations. The violations are very high, so DMPC would need to be tuned to better meet the constraints. This could be achieved by a tightening of the comfort band, see [30]. Similarly, SMPC can be tuned. This is however much easier, as there exists a natural tuning parameter, the violation level $\alpha$. Without any tuning, for all cases, SMPC uses the least amount of NRPE and in four of six cases also the least violations.

\section{CONCLUSION}

A stochastic MPC formulation involving chance constraints which is tractable for large-scale systems such as buildings was presented. This formulation combines the use of ADF with a tractable reformulation of the chance constraints. Three reformulations were compared and analyzed on a small-scale example. One formulation, which turned out to be well-suited for the purpose of building control was tested on a large-scale building example and compared to current control practice as well as a theoretical benchmark.

\section{APPENDIX I}

To use the results from [21], the argument of (11) is equivalently restated as

$$
\begin{aligned}
\left(\left[\mathbf{G}_{j} \mathbf{B} \mathbf{0}_{1 \times n_{u} n_{p} N^{2}}\right]+\right. & {\left.\left[\mathbf{0}_{1 \times n_{u} N}\left(\Xi_{j} \mathbf{w}\right)^{T}\right]\right) \mathbf{z} } \\
& +\left(\mathbf{G}_{j} \mathbf{A} x_{0}-\mathbf{g}_{j}+\mathbf{G}_{j} \mathbf{E w}\right) \leq 0
\end{aligned}
$$

where $\mathbf{z}:=\left[\mathbf{h}^{T} \mathbf{m}^{T}\right]^{T}$ contains the vectors $\mathbf{h}$ and $\mathbf{m}$, where $\mathbf{m}$ is a vectorized version of $\mathbf{M}$ defined as

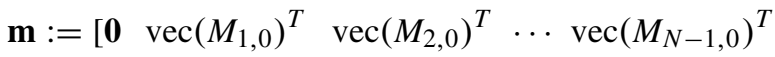

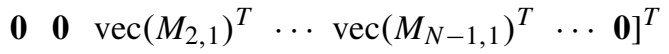


and $\Xi_{j}$ is defined as follows. To show equivalence of (11) and (21), the only nonobvious part is to show that $\mathbf{G}_{j} \mathbf{B M w}=$ $\left(\Xi_{j} \mathbf{w}\right)^{T} \mathbf{m}$. First, note that $\mathbf{G}_{j} \mathbf{B M w}$ is scalar $\Rightarrow \mathbf{G}_{j} \mathbf{B M w}=$ $\left(\mathbf{G}_{j} \mathbf{B M w}\right)^{T}$. Similarly, $\left(\Xi_{j} \mathbf{w}\right)^{T} \mathbf{m}=\mathbf{m}^{T} \Xi_{j} \mathbf{w}$. Thus, it needs to be shown that $\mathbf{G}_{j} \mathbf{B M}=\mathbf{m}^{T} \Xi_{j}$.

Let

$$
f^{T}:=\mathbf{G}_{j} \mathbf{B}
$$

with

$$
f^{T}=\left[f_{1} \ldots f_{N}\right] . \Rightarrow \mathbf{G}_{j} \mathbf{B M}=f^{T} \mathbf{M} .
$$

For each $j \in \mathbb{N}_{0}^{N-1}$ it can be written

$$
f_{j} M_{k, l}=\operatorname{vec}\left(M_{k, l}\right)^{T}\left(\mathbf{I}_{m p} \otimes f_{j}\right) .
$$

Define

$$
\hat{F}^{j}:=\left[\mathbf{I}_{m p} \otimes f_{0} \mathbf{I}_{m p} \otimes f_{1} \ldots\right]^{T}
$$

which multiplies each column of M. Consequently, if $\Xi_{j}:=$ $\left(\mathbf{I} \otimes \hat{F}^{j}\right), f^{T} \mathbf{M}$ can be written as $\mathbf{m}^{T} \Xi_{j}$. Having the inequality in this form, the assertion now follows from [21, Th. 2b].

\section{ACKNOWLEDGMENT}

The authors gratefully acknowledge the contributions of the OptiControl project participants for the large-scale building control example (www.opticontrol.ethz.ch).

\section{REFERENCES}

[1] Indoor Environmental Input Parameters for Design and Assessment of Energy Performance of Buildings Addressing Indoor Air Quality, Thermal Environment, Lighting and Acoustics, Standard En 15251:2007, 2008.

[2] F. Oldewurtel, A. Parisio, C. Jones, D. Gyalistras, M. Gwerder, V. Stauch, B. Lehmann, and M. Morari, "Use of model predictive control and weather predictions for energy efficient building climate control," Energy Buildings, vol. 45, pp. 15-27, Feb. 2012.

[3] Y. Ma, F. Borrelli, B. Hencey, B. Coffey, S. Bengea, and P. Haves, "Model predictive control for the operation of building cooling systems," IEEE Trans. Control Syst. Technol., vol. 20, no. 3, pp. 796-803, May 2012.

[4] J. Siroky, F. Oldewurtel, J. Cigler, and S. Privara, "Experimental analysis of model predictive control for an energy efficient building heating system," Appl. Energy, vol. 88, no. 9, pp. 3079-3087, 2011.

[5] G. Henze, C. Felsmann, and G. Knabe, "Impact of forecasting accuracy on predictive optimal control of active and passive building thermal storage inventory," Int. J. HVAC\&R Res., vol. 10, no. 2, pp. 153-178, 2004.

[6] V. Zavala, "Real-time optimization strategies for building systems," Ind. Eng. Chem. Res., vol. 52, no. 9, pp. 3137-3150, 2013.

[7] T. Salsbury, P. Mhaskar, and S. Qin, "Predictive control methods to improve energy efficiency and reduce demand in buildings," Comput. Chem. Eng., vol. 51, pp. 77-85, Apr. 2013.

[8] A. Aswani, N. Master, J. Taneja, D. Culler, and C. Tomlin, "Reducing transient and steady state electricity consumption in hvac using learning-based model predictive control," Proc. IEEE, vol. 100, no. 1, pp. 240-253, Jan. 2012.

[9] S. Goyal, H. Ingley, and P. Barooah, "Zone-level control algorithms based on occupancy information for energy efficient buildings," in Proc. Amer. Control Conf., Jun. 2012, pp. 3063-3068.

[10] A. Mady, G. Provan, C. Ryan, and K. Brown, "Stochastic model predictive controller for the integration of building use and temperature regulation," in Proc. 25th AAAI Conf. Artif. Intell., 2011, pp. 1371-1376.

[11] (2011, Jul. 28). OptiControl Project [Online]. Available: http://www.opticontrol.ethz.ch

[12] F. Oldewurtel, C. Jones, and M. Morari, "A tractable approximation of chance constrained stochastic MPC based on affine disturbance feedback," in Proc. IEEE CDC, Dec. 2008, pp. 4731-4736.
[13] A. Bemporad, "Reducing conservativeness in predictive control of constrained systems with disturbances," in Proc. 37th IEEE Conf. Decision Control, Dec. 1998, pp. 1384-1391.

[14] L. Chisci, J. Rossiter, and G. Zappa, "Systems with persistent state disturbances: Predictive control with restricted constraints," Automatica, vol. 37, no. 7, pp. 1019-1028, 2001.

[15] E. Kerrigan and J. Maciejowski, "On robust optimization and the optimal control of constrained linear systems with bounded state disturbances," in Proc. Eur. Control Conf., 2003, pp. 1-6.

[16] A. Ben-Tal, A. Goryashko, E. Guslitzer, and A. Nemirovski, "Adjustable robust solutions of uncertain linear programs," Math. Program., vol. 99, pp. 351-376, Mar. 2004.

[17] J. Löfberg, "Approximation of closed-loop minimax MPC," in Proc. 42nd IEEE Conf. Decision Control, Dec. 2003, pp. $1438-1442$.

[18] D. H. van Hessem and O. Bosgra, "A conic reformulation of model predictive control including bounded and stochastic disturbances under state and input constraints," in Proc. 41st IEEE Conf. Decision Control, Dec. 2002, pp. 4643-4648.

[19] P. Goulart, E. Kerrigan, and J. Maciejowski, "Optimization over state feedback policies for robust control with constraints," Automatica, vol. 42 , no. 4 , pp. $523-533,2006$.

[20] P. Hokayem, D. Chatterjee, and J. Lygeros, "On stochastic receding horizon control with bounded control inputs," in Proc. Conf. Decision Control, 2009, pp. 6359-6364.

[21] D. Bertsimas and M. Sim, "Tractable approximations to robust conic optimization problems," Math. Program, vol. 107, nos. 1-2, pp. 5-36, 2006.

[22] A. Charnes, W. Cooper, and G. Symonds, "Cost horizons and certainty equivalents: An approach to stochastic programming of heating oil," Manag. Sci., vol. 4, no. 3, pp. 235-263, 1958.

[23] A. Prekopa, Stochastic Programming. Dordrecht, The Netherlands: Kluwer, 1995.

[24] P. Kall and J. Mayer, Stochastic Linear Programming. Boston, MA, USA: Kluwer, 2005.

[25] J. Birge and F. Louveaux, Introduction to Stochastic Programming (Operations Research). New York, NY, USA: Springer-Verlag, 1997.

[26] D. Bernardini and A. Bemporad, "Scenario-based model predictive control of stochastic constrained linear systems," in Proc. 48th IEEE CDC/CCC, Dec. 2009, pp. 6333-6338.

[27] M. Cannon, B. Kouvaritakis, S. Rakovic, and Q. Chen, "Stochastic tubes in model predictive control with probabilistic constraints," in Proc. Amer. Control Conf., Jun./Jul. 2010, pp. 6274-6279.

[28] Y. Wang and S. Boyd, "Performance bounds for linear stochastic control," Syst. Control Lett., no. 58, no. 3, pp. 178-182, 2009.

[29] M. Gwerder and J. Tödli, "Predictive control for integrated room automation," in Proc. 8th REHVA World Congr. Building Technol., Oct. 2005, pp. 1-6.

[30] F. Oldewurtel, A. Parisio, C. Jones, M. Morari, D. Gyalistras, M. Gwerder, V. Stauch, B. Lehmann, and K. Wirth, "Energy efficient building climate control using stochastic model predictive control and weather predictions," in Proc. Amer. Control Conf., Jun./Jul. 2010, pp. $5100-5105$

[31] D. Gyalistras and M. Gwerder, "Use of weather and occupancy forecasts for optimal building climate control (opticontrol): Two years progress report," ETH Zurich and Siemens Building Technologies Division, Siemens Switzerland Ltd., Zug, Switzerland, Tech. Rep., 2009 [Online]. Available: http://www.opticontrol.ethz.ch/Lit/Gyal_10_OptiControl2YearsReport. pdf

[32] V. Stauch, F. Schubiger, and P. Steiner, "Local weather forecasts and observations," ETH Zurich and Siemens Building Technologies Division, Siemens Switzerland Ltd., Zug, Switzerland, Tech. Rep., 2009 [Online]. Available: www.opticontrol.ethz.ch/Lit/Gyal_10_OptiControl2YearsReport.pdf.

[33] R. Griffith and R. Steward, "A nonlinear programming technique for the optimization of continuous processing systems," J. Manag. Sci., vol. 7, no. 4, pp. 379-392, 1961.

[34] M. Gwerder, J. Tödtli, and D. Gyalistras, "Rule-based control strategies," ETH Zurich and Siemens Building Technologies Division, Siemens Switzerland Ltd., Zug, Switzerland, Tech. Rep., 2009 [Online]. Available: www.opticontrol.ethz.ch/Lit/Gyal_10_OptiControl2YearsReport.pdf.

[35] E. Kerrigan, "Robust constraint satisfaction: Invariant sets and predictive control," Ph.D. dissertation, Dep. Eng., Univ. Cambridge, Cambridge, U.K., 2000. 